\title{
Acute Uric Acid Nephropathy
}

National Cancer Institute

\section{Source}

National Cancer Institute. Acute Uric Acid Nephropathy. NCI Thesaurus. Code C123024.

Kidney damage resulting from uric acid precipitation within the renal tubules. 